Journal Club

Editor's Note: These short, critical reviews of recent papers in the Journal, written exclusively by graduate students or postdoctoral fellows, are intended to summarize the important findings of the paper and provide additional insight and commentary. For more information on the format and purpose of the Journal Club, please see http://www.jneurosci.org/misc/ifa_features.shtml.

\title{
A Unique Mechanism of NMDA Spike Initiation Supports a Distinct Role in Synaptic Input Integration
}

\author{
Kevin F. H. Lee \\ Neuroscience Graduate Program, Department of Cellular and Molecular Medicine, Faculty of Medicine, University of Ottawa, Ottawa, Ontario, \\ Canada K1H 8M5 \\ Review of Chalifoux and Carter
}

To maintain the fidelity of information flow in the brain, neurons must integrate and transform complex patterns of synaptic input into distinct patterns of action potential output. These computations are fundamental to neuronal function and depend on the spatial and temporal characteristics of the incoming synaptic activity. Because the dendritic arbors of cortical pyramidal cells can reach hundreds of micrometers, passive dendritic cable properties can strongly attenuate throughput of distal synaptic information to the soma. However, neurons have adopted strategies for boosting the transmission of distal synaptic information. Temporally concerted and spatially clustered synaptic inputs can generate highamplitude regenerative events in individual dendrites called "dendritic spikes" and "NMDA spikes" (London and Häusser, 2005; Larkum and Nevian, 2008). Dendritic spikes occur in apical dendrites of cortical pyramidal neurons and cerebellar Purkinje cells and require the activation of voltagegated $\mathrm{Na}^{+}$and $\mathrm{Ca}^{2+}$ channels (VGSCs and VGCCs, respectively). NMDA spikes take place in the thin, basal, oblique, and apical tuft dendrites of pyramidal neurons and re-

Received Dec. 19, 2011; revised Jan. 13, 2012; accepted Jan. 13, 2012.

The host lab is supported by the Natural Sciences and Engineering Research Council of Canada and the Canadian Institutes of Health Research.

Correspondence should be addressed to Kevin F. H. Lee, Neuroscience Graduate Program, Department of Cellular and Molecular Medicine, Faculty of Medicine, University of Ottawa, 451 Smyth Road, Ottawa, Ontario, Canada K1H 8M5. E-mail: kevin.fh.lee@gmail.com.

DOI:10.1523/JNEUROSCI.6318-11.2012

Copyright $\odot 2012$ the authors $\quad 0270-6474 / 12 / 322913-02 \$ 15.00 / 0$ quire the activation of the NMDA subtype of glutamate receptor (NMDARs), but not VGSCs or VGCCs. Dendritic spikes and NMDA spikes provide robust supralinear synaptic integration, and thus have an important role in neuronal information processing (London and Häusser, 2005; Larkum and Nevian, 2008).

The initiation of dendritic spikes can be ascribed to the simple summation of EPSPs, which provides the depolarization necessary to engage VGSCs and VGCCs. But how brief bursts of synaptic input result in the regenerative activation of NMDARs to initiate NMDA spikes is largely unknown. In principle, the prolonged depolarizations resulting from the slow kinetics of NMDAR deactivation/inactivation may facilitate the activation of additional NMDARs upon subsequent synaptic activation by providing sustained relief of the voltagedependent magnesium block (Polsky et al., 2009). However, long-decaying depolarization is believed to be incapable of providing the high conductance required to elicit NMDA spikes (Rhodes, 2006). Furthermore, membrane depolarization alone is insufficient for NMDA spike generation (Polsky et al., 2009). An intriguing alternative is that glutamate released during highfrequency synaptic activation might result in glutamate spillover and the subsequent activation of additional, nonsynaptic populations of NMDARs. In a recent issue of The Journal of Neuroscience, Chalifoux and Carter (2011) investigated this possibility by visualizing NMDAR spike-mediated cal- cium transients via two-photon calcium imaging in the basal dendrites of layer $\mathrm{V}$ pyramidal neurons from mouse prefrontal cortex.

Using low stimulus intensities, Chalifoux and Carter (2011) evoked archetypal NMDA spikes by paired-pulse stimulation at $50 \mathrm{~Hz}$. As expected, generation of NMDA spikes required activation of both AMPARs and NMDARs and resulted in large calcium transients that invaded adjacent dendrites and spines. Blocking Tand L-type VGCCs and depleting internal $\mathrm{Ca}^{2+}$ stores did not affect NMDA spike calcium dynamics, ruling out the contribution of these calcium sources. Together, the data convincingly identify NMDARs as the primary source of calcium influx during NMDA spikes.

Interestingly, increasing the number of stimuli produced monotonically larger $\mathrm{Ca}^{2+}$ transients in neighboring spine and dendrite compartments, an effect that was enhanced by blocking glutamate transporters with a low concentration of threo- $\beta$-benzyloxyaspartic acid. This finding suggested that the generation of NMDA spikes involved glutamate spillover and activation of surrounding nonsynaptic NMDAR populations. As a mechanism for NMDA spike initiation, glutamate spillover accentuates the highly local nature of this dendritic supralinearity and underscores the different roles of dendritic spikes and NMDA spikes in synaptic integration.

Brief trains of high-frequency stimuli can evoke both dendritic spikes and NMDA spikes (Golding and Spruston, 1998; Schiller 
et al., 2000). However, the differences in subcellular location and mechanism of initiation (dependence on VGCCs/VGSCs vs NMDARs) indicates an important distinction in the spatial rules that govern the generation of these events, and also hints at the fundamentally distinct roles they might play in neuronal information processing. Dendritic spikes can be generated by spatially disparate synaptic inputs due to the pure voltage dependence of initiation-a behavior that is highly analogous to the threshold-based discharge of action potentials at the axon initial segment. In contrast, the necessity for glutamate spillover and NMDAR activation imparts a spatial limitation on NMDA spike generation, restricting these events to the individual dendritic branch receiving the burst of synaptic input. Recently, dendritic spikes and NMDA spikes were proposed to perform distinct roles in a multilayered model of dendritic integration (Larkum et al., 2009). In this model, spatially clustered bursts of synaptic inputs in thin distal dendrites initiate NMDA spikes, which propagate toward increasingly largercaliber dendrites en route to the soma. At these larger dendrites, converging depolarizations (perhaps multiple NMDA spikes from different dendrites) can temporally summate to initiate dendritic spikes, which ultimately amplify these events to influence action potential firing at the soma. This multilayered integration process provides a conceptual framework that encompasses the relative roles of dendritic and NMDA spikes in boosting the throughput of temporally synchronous synaptic events. Together, these distinct events provide neurons with the ability to robustly enhance the signal-tonoise ratio of coherent synaptic information while ignoring uncoordinated synaptic noise.

Cortical neurons often communicate via bursts of action potentials, and as few as two or three afferent action potentials can be sufficient to drive postsynaptic NMDA spikes (Polsky et al., 2009). NMDA spikes can thus provide an online readout of local burst input, offering clear theoretical advantages to synaptic integration and information throughput. However, the novel role of glutamate spillover in the generation of NMDA spikes raises an important question: does glutamate spillover occur at all synapses in the brain? Synapses are conventionally regarded as isolated functional units that relay discrete point-to-point synaptic information. This functional isolation is well appreciated in light of the apparent synapse-specificity of synaptic plasticity and the functional autonomy of synapses in theoretical models of information storage and learning. The occurrence of glutamate spillover suggests that the mechanisms that regulate extracellular glutamate concentrations (notably, neuronal and glial glutamate transporters) become saturated during bursts of synaptic activity, introducing a degree of synaptic promiscuity that challenges the point-to-point specificity of synaptic transmission. Because NMDA spikes have been identified primarily in thin dendrites of cortical and hippocampal pyramidal neurons, it is possible that the expression of glutamate transport mechanisms are differentially regulated in a dendrite-specific manner. In principle, this would provide a novel role for glutamate clearance mechanisms in the regulation of dendritic excitability. Speculation aside, it is important to consider that standard electrophysiological methods involving high-frequency stimuli (paired-pulse stimulation, LTP-induction) may be confounded by glutamate spillover and the activation of nonsynaptic glutamate receptors. Thus, it will be important for future studies to resolve the spatiotemporal characteristics and the pervasiveness of glutamate spillover at cortical and hippocampal synapses.

Neurophysiologists have developed a growing appreciation for the spatially compartmentalized nature of synaptic integration, dendritic excitability, and synaptic plasticity. The data provided by Chalifoux and Carter (2011) further champions these ideas. The novel role of glutamate spillover in NMDA spike initiation accentuates the highly local nature of these events and further distinguishes them from dendritic spikes. The apparent subcellular segregation of dendritic and NMDA spikes supports a recently proposed multilayer model of dendritic integration, providing a conceptual framework for the relative roles of these regenerative events in neuronal information processing (Larkum et al., 2009). At the same time, the data provided by Chalifoux and Carter (2011) have important implications for synaptic physiology in general. It will be interesting to see how future studies of glutamate spillover impact our understanding of synaptic and dendritic function, and the broader implications they impress upon neuronal communications and information processing.

\section{References}

Chalifoux JR, Carter AG (2011) Glutamate spillover promotes the generation of NMDA spikes. J Neurosci 31:16435-16446.

Golding NL, Spruston N (1998) Dendritic sodium spikes are variable triggers of axonal action potentials in hippocampal CA1 pyramidal neurons. Neuron 21:1189-1200.

Larkum ME, Nevian T (2008) Synaptic clustering by dendritic signalling mechanisms. Curr Opin Neurobiol 18:321-331.

Larkum ME, Nevian T, Sandler M, Polsky A, Schiller J (2009) Synaptic integration in tuft dendrites of layer 5 pyramidal neurons: a new unifying principle. Science 325:756-760.

London M, Häusser M (2005) Dendritic computation. Annu Rev Neurosci 28:503-532.

Polsky A, Mel B, Schiller J (2009) Encoding and decoding bursts by NMDA spikes in basal dendrites of layer 5 pyramidal neurons. J Neurosci 29:11891-11903.

Rhodes P (2006) The properties and implications of NMDA spikes in neocortical pyramidal cells. J Neurosci 26:6704-6715.

Schiller J, Major G, Koester HJ, Schiller Y (2000) NMDA spikes in basal dendrites of cortical pyramidal neurons. Nature 404:285-289. 